\title{
FUNCTIONAL REALIZATIONS OF LIE ALGEBRAS AS NOETHER POINT SYMMETRIES OF SYSTEMS
}

\author{
Rutwig Campoamor-Stursberg
}

\author{
Instituto de Matemática Interdisciplinar-UCM, Plaza de Ciencias 3, E-28040 Madrid, Spain \\ correspondence: rutwig@ucm.es
}

\begin{abstract}
Functional realizations of Lie algebras are applied to the problem of determining Lie and Noether point symmetries of Lagrangian systems in $N$ dimensions, particularly in the plane. This encompasses both the case of symmetry-preserving perturbations of a given system, as well as the generic analysis on the structure of (regular) Lagrangians in order to admit a symmetry algebra belonging to a specific isomorphy class.
\end{abstract}

KEYWORDS: Lagrangian; Lie point symmetry; Noether symmetry; perturbation.

\section{INTRODUCTION}

The study of the precise relation between constants of the motion of a dynamical system and the symmetry properties of the corresponding equations of the motion (alternatively, an associated Lagrangian) goes back to the seminal paper of E. Noether [1], a key result that, combined with the Lie group theoretical approach to differential equations, has become an essential tool in many branches of applied mathematics and Mechanics (see, e.g., 2, 3, and references therein).

Usually, the Noether symmetry analysis is carried out mainly for conservative systems, due to requirements of the Hamiltonian formalism and the corresponding quantization of the systems 4. However, there is no restriction, at least from the Lie algebraic point of view, to treat both conservative and dissipative systems simultaneously. This can be done introducing appropriate functional realizations of Lie algebras, the symmetry generators of which depend on the coordinates of the extended configuration space, and considering the constraints imposed by this timedependence. This extended approach allows to cover physically relevant systems, such as time-modulated oscillators, and has further applications in the context of perturbation theory, such as the study of some geometric properties of the orbits of a given system [5].

Within the context of inverse of problems in dynamics [5, 6], in this work we consider an inverse approach to dynamics based on functional realizations of Lie algebras that are required to satisfy the Noether symmetry condition for a (regular) Lagrangian system. This allows two approaches, either starting from a given system and analyzing perturbations that preserve certain of the symmetries, or determining families of systems invariant with respect to these realizations. For the purpose of illustration, the plane case is considered, although the Ansatz can also be formulated in arbitrary dimensions.

\section{LIE AND NOETHER POINT SYMMETRIES OF SYSTEMS}

Let $L(t, \mathbf{q}, \dot{\mathbf{q}})$ be a regular Lagrangian in $n$-dimensions and let

$$
\frac{d}{d t}\left(\frac{\partial L}{\partial \dot{q}^{i}}\right)-\frac{\partial L}{\partial q^{i}}=0, \quad 1 \leq i \leq n
$$

be the corresponding equations of the motion. By regularity, we can always rewrite the equations of motion in normal form, i.e.,

$$
\ddot{q}^{i}=\omega_{i}\left(t, \dot{q}^{j}, q^{j}\right)=g^{i j}\left(\frac{\partial L}{\partial q^{j}}-\frac{\partial^{2} L}{\partial t \partial \dot{q}^{j}}-\frac{\partial^{2} L}{\partial \dot{q}^{j} \partial q^{k}} \dot{q}^{k}\right),
$$

for $1 \leq i \leq n$, with $g^{i j}$ denoting the inverse Hessian matrix of $L$. As is well known, the system (2) can be reformulated in equivalent form as the first order partial differential equation

$$
\mathbf{A} f=\left(\frac{\partial}{\partial t}+\dot{q}^{i} \frac{\partial}{\partial q^{i}}+\omega_{i} \frac{\partial}{\partial \dot{q}^{i}}\right) f=0 .
$$

We call a vector field $\mathbf{X}=\xi(t, \mathbf{q}) \frac{\partial}{\partial t}+\eta_{j}(t, \mathbf{q}) \frac{\partial}{\partial q_{j}} \in$ $\mathfrak{X}\left(\mathbb{R}^{N+1}\right)$ a Lie point symmetry of the equations $(2)$ if its first prolongation $\dot{\mathbf{X}}=\mathbf{X}+\dot{\eta}_{j}(t, \mathbf{q}, \dot{\mathbf{q}}) \frac{\partial}{\partial \dot{q}_{j}}$ with $\dot{\eta}_{j}=-\frac{d \xi}{d t} \dot{q}_{j}+\frac{d \eta_{j}}{d t}$ satisfies the commutator

$$
[\dot{\mathbf{X}}, \mathbf{A}]=-\frac{d \xi}{d t} \mathbf{A}
$$

Lie point symmetries span a finite-dimensional Lie algebra $\mathcal{L}_{P S}$, called the Lie (point) symmetry algebra of the system, that be seen to be always a subalgebra of the simple algebra $\mathfrak{s l}(n+2, \mathbb{R})$, corresponding to the free system $\ddot{\mathbf{q}}=0$ [7, 8 .

Whenever the system arises from a Lagrangian, a constant of the motion of (2) is defined as a function $F(t, \mathbf{q}, \dot{\mathbf{q}})$ satisfying the condition

$$
\frac{d F}{d t}=\frac{\partial F}{\partial t}+\dot{q}^{i} \frac{\partial F}{\partial q^{i}}+\ddot{q}^{i} \frac{\partial F}{\partial \dot{q}^{i}}=\mathbf{A}(F)=0 .
$$


In the following, we will mainly consider Lie point and Noether point symmetries and the conservation laws associated to them.

In this context, a vector field

$$
\mathbf{X}=\xi(t, \mathbf{q}) \frac{\partial}{\partial t}+\eta^{j}(t, \mathbf{q}) \frac{\partial}{\partial q^{j}}
$$

is called a Noether point symmetry of (2) if it satisfies the constraint

$$
\dot{\mathbf{X}}(L)+\mathbf{A}(\xi) L-\mathbf{A}(V)=0,
$$

for some function $V(t, \mathbf{q})$ that does not depend on the velocities, and that we usually refer to as the gauge term of the symmetry generator. Expanding the symmetry condition (7) provides the following partial differential equation

$$
\begin{aligned}
\xi(t, \mathbf{q}) \frac{\partial L}{\partial t}+\eta^{j} & (t, \mathbf{q}) \frac{\partial L}{\partial q^{j}}+\dot{\eta}^{j}(t, \mathbf{q}, \dot{\mathbf{q}}) \frac{\partial L}{\partial \dot{q}^{j}}+ \\
& \frac{d \xi}{d t} L(t, \mathbf{q}, \dot{\mathbf{q}})-\frac{\partial V}{\partial t}-\dot{q}^{j} \frac{\partial V}{\partial q^{j}}=0 .
\end{aligned}
$$

The first Noether theorem (see e.g. [1]) states that to any symmetry (6) there corresponds a constant of the motion determined by the rule

$$
J=\xi\left(\dot{q}^{k} \frac{\partial L}{\partial \dot{q}^{k}}-L\right)-\eta^{k} \frac{\partial L}{\partial \dot{q}^{k}}+V(t, \mathbf{q}) .
$$

It follows in particular that a constant of the motion $J$ is an invariant of $\dot{\mathbf{X}}$, i.e., the condition $\dot{\mathbf{X}}(J)=0$ is satisfied.

\subsection{Perturbations that PRESERVE SYMMETRY SUBALGEBRAS}

Given a system described by a Lagrangian $L(t, \mathbf{q}, \dot{\mathbf{q}})$ and having an $r$-dimensional Lie algebra $\mathcal{L}_{N S}$ of Noether point symmetries, fixing a certain subalgebra $\mathcal{L}_{0}<\mathcal{L}_{N S}$ of dimension $r_{0}<r$ we can ask whether a perturbed Lagrangian $\widehat{L}=L+\varepsilon S(t, \mathbf{q}, \dot{\mathbf{q}})$ exists such that the symmetry generators $\mathbf{X}_{j}\left(1 \leq j \leq r_{0}\right)$ of $\mathcal{L}_{0}$ are still Noether point symmetries of $\widehat{L}$. Clearly, the equations of the motion $\sqrt{1}$ of $\widehat{L}$ are given by

$$
\frac{d}{d t}\left(\frac{\partial L}{\partial \dot{q}^{i}}\right)-\frac{\partial L}{\partial q^{i}}+\frac{d}{d t}\left(\frac{\partial S}{\partial \dot{q}^{i}}\right)-\frac{\partial S}{\partial q^{i}}=0,
$$

so that the symmetry condition for the subalgebra $\mathcal{L}_{0}$ leads, for each generator $\mathbf{X}_{k}\left(1 \leq k \leq r_{0}\right)$, to the differential equation

$$
\begin{aligned}
& \dot{\mathbf{X}}_{k}(\widehat{L})+\mathbf{A}\left(\xi_{k}\right) \widehat{L}-\mathbf{A}\left(V_{k}\right)=\dot{\mathbf{X}}_{k}(L) \\
& \quad+\mathbf{A}\left(\xi_{k}\right) L-\mathbf{A}\left(V_{k}\right)+\dot{\mathbf{X}}_{k}(S)+\frac{d \xi_{k}}{d t} S=0 .
\end{aligned}
$$

Now the vector fields $\mathbf{X}_{k}$ are Noether symmetries of the Lagrangian $L$, and fixing the gauge terms $V_{k}(t, \mathbf{q})$, the equation (10) simplifies to a PDE involving merely the perturbation term $S(t, \mathbf{q}, \dot{\mathbf{q}})$ :

$$
\begin{aligned}
\dot{\mathbf{X}}_{k}(S)+ & \frac{d \xi_{k}}{d t} S(t, \mathbf{q}, \dot{\mathbf{q}})=\xi_{k}(t, \mathbf{q}) \frac{\partial S}{\partial t}+\eta_{k}^{j}(t, \mathbf{q}) \frac{\partial S}{\partial q^{j}} \\
& +\dot{\eta}_{k}^{j}(t, \mathbf{q}, \dot{\mathbf{q}}) \frac{\partial S}{\partial \dot{q}^{j}}+\frac{d \xi_{k}}{d t} S(t, \mathbf{q}, \dot{\mathbf{q}})=0, \quad(11)
\end{aligned}
$$

where $1 \leq k \leq r_{0}$. In the case of velocity-independent perturbation terms, a straightforward verification shows that a Noether symmetry is preserved only if $\frac{\partial \xi_{k}}{\partial q^{j}}=0$ holds for all $1 \leq k \leq r_{0}$ and $1 \leq j \leq n$. This allows to restrict the perturbation analysis to subalgebras $\mathcal{L}_{0}$, the generators of which have components of the type $\xi_{k}=\varphi_{k}(t)$. This agrees with the usually observed pattern of Lie point symmetries of nonlinear second-order systems of differential equations of the type $\ddot{\mathbf{q}}=\omega(t, \mathbf{q})[7,8]$.

\section{FunCTIONAL REALIZATIONS OF $\mathfrak{s l}(2, \mathbb{R})$ AS NOETHER SYMMETRY ALGEBRA}

As has been already pointed out in many contexts, the non-compact Lie algebra $\mathfrak{s l}(2, \mathbb{R})$ plays a relevant role within the group-theoretic analysis of differential equations, in particular, concerning the (super)integrability of plane systems and the linearization analysis 911. This fact suggests to consider this Lie algebra more closely in the context of inverse problems, as done in [12]. For these reasons, in the following we restrict our analysis to $\mathfrak{s l}(2, \mathbb{R})$.

Prior to analyzing a functional realization of $\mathfrak{s l}(2, \mathbb{R})$, we make some observations on the generic structure of Noether point symmetries. Consider to this extent a Lagrangian adopting the kinetic form

$$
T=\frac{1}{2} A_{i j}(\mathbf{q}) \dot{q}_{i} \dot{q}_{j},
$$

such that $A_{11}(\mathbf{q}) A_{22}(\mathbf{q})-A_{12}(\mathbf{q})^{2} \neq 0$ holds.

Lemma 1. Let $\mathbf{X}=\xi(t, \mathbf{q}) \frac{\partial}{\partial t}+\eta_{j}(t, \mathbf{q}) \frac{\partial}{\partial q_{j}}$ be a Noether point symmetry of a Lagrangian (12). Then the components have the generic structure

$$
\begin{gathered}
\xi(t, \mathbf{q})=C_{1} t^{2}+C_{2} t+C_{3}, \\
\eta_{j}(t, \mathbf{q})=\eta_{j 1}(\mathbf{q}) t+\eta_{j 2}(\mathbf{q}) .
\end{gathered}
$$

Moreover, the gauge term does not explicitly depend of time, i.e., $V=V(\mathbf{q})$.

Proof. Developing the symmetry condition (7) and keeping only the independent term, as well as the terms with highest power in $\mathbf{q}$, we obtain that $\frac{\partial V}{\partial t}=0$ and the conditions

$$
\begin{gathered}
\frac{\partial \xi}{\partial q_{1}} A_{11}(\mathbf{q})=0, \quad \frac{\partial \xi}{\partial q_{2}} A_{22}(\mathbf{q})=0 \\
\frac{\partial \xi}{\partial q_{2}} A_{12}(\mathbf{q})+\frac{1}{2} \frac{\partial \xi}{\partial q_{1}} A_{22}(\mathbf{q})=0 \\
\frac{\partial \xi}{\partial q_{1}} A_{12}(\mathbf{q})+\frac{1}{2} \frac{\partial \xi}{\partial q_{2}} A_{22} \cdot(\mathbf{q})=0
\end{gathered}
$$


As $A_{11}(\mathbf{q}) A_{22}(\mathbf{q})-A_{12}(\mathbf{q})^{2} \neq 0$, a short computation shows that the condition $\frac{\partial \xi}{\partial q_{1}}=\frac{\partial \xi}{\partial q_{2}}=0$ must be necessarily satisfied. Introducing this into the symmetry condition, the following relations are obtained for the terms linear in $\mathbf{q}$ :

$$
\begin{aligned}
& \frac{\partial \eta_{2}}{\partial t} A_{12}(\mathbf{q})+\frac{\partial \eta_{1}}{\partial t} A_{11}(\mathbf{q})-\frac{\partial V}{\partial q_{1}}=0 \\
& \frac{\partial \eta_{1}}{\partial t} A_{12}(\mathbf{q})+\frac{\partial \eta_{2}}{\partial t} A_{22}(\mathbf{q})-\frac{\partial V}{\partial q_{2}}=0 .
\end{aligned}
$$

Multiplying the first equation by $A_{12}$, the second by $-A_{11}$ and adding them leads to the expression

$$
\frac{\partial \eta_{2}}{\partial t}\left(A_{12}^{2}-A_{11} A_{22}\right)+A_{12} \frac{\partial V}{\partial q_{1}}+A_{11} \frac{\partial V}{\partial q_{2}}=0,
$$

from which we conclude that $\eta_{2}$ is at most linear in $t$, hence it admits a decomposition $\eta_{2}(t, \mathbf{q})=\eta_{21}(\mathbf{q}) t+$ $\eta_{22}(\mathbf{q})$. For $\eta_{1}(t, \mathbf{q})$ the assertion is obtained similarly. Finally, for the terms quadratic in $\mathbf{q}$ in the symmetry condition (7), we have expression of the type

$$
-\frac{1}{2} A_{i j}(\mathbf{q}) \frac{d \xi}{d t}+t \Psi_{1}(\mathbf{q})+\Psi_{2}(\mathbf{q})=0,
$$

showing that $\xi(t)$ is at most quadratic in $t$, from which the assertion follows.

We observe that the generalization of the latter result to $n$-dimensions is straightforward.

Now let $f(\mathbf{q})$ and $g(\mathbf{q})$ be arbitrary non-vanishing functions and consider the Lie algebra generated by the following vector fields:

$$
\begin{gathered}
X_{1}=t^{2} \frac{\partial}{\partial t}+t f(\mathbf{q}) \frac{\partial}{\partial q_{1}}+t g(\mathbf{q}) \frac{\partial}{\partial q_{2}} \\
X_{2}=\frac{1}{2} \frac{d}{d t} X_{1}=t \frac{\partial}{\partial t}+\frac{1}{2} f(\mathbf{q}) \frac{\partial}{\partial q_{1}}+\frac{1}{2} g(\mathbf{q}) \frac{\partial}{\partial q_{2}}, \\
X_{3}=\frac{1}{2} \frac{d^{2}}{d t^{2}} X_{1}=\frac{\partial}{\partial t} .
\end{gathered}
$$

It follows at once that $\left[X_{1}, X_{2}\right]=-X_{1},\left[X_{1}, X_{3}\right]=$ $-2 X_{2}$ and $\left[X_{2}, X_{3}\right]=-X_{3}$, showing that the Lie algebra is isomorphic to $\mathfrak{s l}(2, \mathbb{R})$ for any choices of $f$ and $g$. We observe that the structure of the $\mathfrak{s l}(2, \mathbb{R})$ Lie algebra generalizes naturally that studied in [8, 12]. In these conditions, it can be asked which is the most general (kinetic) Lagrangian such that it admits this Lie algebra as an algebra of Noether point symmetries. It suffices to impose the invariance with respect to $X_{1}$ and $X_{3}$ in order to ensure that the system is $\mathfrak{s l}(2, \mathbb{R})$ invariant. The symmetry condition (7) applied to $X_{3}$ is trivially satisfied for the gauge term $V(t, \mathbf{q})=0$. Analyzing now the invariance with respect to $X_{1}$, and inspecting first the terms linear in $\dot{\mathbf{q}}$, leads to the constraints

$$
\begin{aligned}
& f(\mathbf{q}) A_{11}(\mathbf{q})+g(\mathbf{q}) A_{12}(\mathbf{q})-\frac{\partial V}{\partial q_{1}}=0 \\
& g(\mathbf{q}) A_{22}(\mathbf{q})+f(\mathbf{q}) A_{12}(\mathbf{q})-\frac{\partial V}{\partial q_{2}}=0
\end{aligned}
$$

for the gauge term $V(\mathbf{q})$. As $f(\mathbf{q}) g(\mathbf{q}) \neq 0$, this allows us to set

$f^{2}(\mathbf{q}) A_{11}(\mathbf{q})-g^{2}(\mathbf{q}) A_{22}(\mathbf{q})-f(\mathbf{q}) \frac{\partial V}{\partial q_{1}}-g(\mathbf{q}) \frac{\partial V}{\partial q_{2}}=0$.

In order to completely satisfy the symmetry condition, the functions $A_{i j}(\mathbf{q})$ must be solutions to the following system of PDEs:

$$
\begin{aligned}
& f(\mathbf{q}) \frac{\partial A_{11}}{\partial q_{1}}+g(\mathbf{q}) \frac{\partial A_{11}}{\partial q_{2}}+2 A_{11}(\mathbf{q})\left(\frac{\partial f}{\partial q_{1}}-1\right) \\
& +2 A_{12}(\mathbf{q}) \frac{\partial g}{\partial q_{1}}=0 \\
& f(\mathbf{q}) \frac{\partial A_{22}}{\partial q_{1}}+g(\mathbf{q}) \frac{\partial A_{22}}{\partial q_{2}}+2 A_{22}(\mathbf{q})\left(\frac{\partial g}{\partial q_{2}}-1\right) \\
& +2 A_{12}(\mathbf{q}) \frac{\partial f}{\partial q_{2}}=0, \\
& f(\mathbf{q}) \frac{\partial A_{12}}{\partial q_{1}}+g(\mathbf{q}) \frac{\partial A_{12}}{\partial q_{2}}+A_{12}(\mathbf{q})\left(\frac{\partial f}{\partial q_{1}}+\frac{\partial g}{\partial q_{2}}-2\right) \\
& +A_{11}(\mathbf{q}) \frac{\partial f}{\partial q_{2}}+A_{22}(\mathbf{q}) \frac{\partial g}{\partial q_{1}}=0 .
\end{aligned}
$$

There are in principle two different ways to analyze these systems related to a Lagrangian of type 12 : either we fix the latter and search for functions $f(\mathbf{q})$ and $g(\mathbf{q})$ such that $(17)$ is a Lie algebra of Noether symmetries, or we fix the components of the symmetry generators and try to determine the most general kinetic term 12 invariant under the algebra. The same problem can be formulated allowing a potential term. We shall exhibit examples of the two approaches leading to nontrivial solutions of the equations.

\subsection{Separable kinetic Lagrangians}

Let us illustrate the preceding situation first for the case of the separable Lagrangians

$$
T=\frac{1}{2}\left(q_{1}^{k} \dot{q}_{1}^{2}+q_{2}^{l} \dot{q}_{2}^{2}\right)
$$

where $k, l \neq-2$ are constants. This case contains in particular that of the free Euclidean Lagrangian, that is well known to allow a $\mathfrak{s l}(2, \mathbb{R})$-subalgebra of Noether symmetries [4. The symmetry condition (*) thus requires the solving for the unknown functions $f(\mathbf{q})$ and $g(\mathbf{q})$, as well as the gauge term $V(\mathbf{q})$ for the symmetry generator $X_{1}$. The resulting equations are

$$
\begin{aligned}
& l g(\mathbf{q})+2 q_{2}\left(\frac{\partial g}{\partial q_{2}}-1\right)=0, \quad q_{2}^{l} g(\mathbf{q})-\frac{\partial V}{\partial q_{2}}=0, \\
& k f(\mathbf{q})+2 q_{1}\left(\frac{\partial f}{\partial q_{1}}-1\right)=0, \quad q_{1}^{l} f(\mathbf{q})-\frac{\partial V}{\partial q_{1}}=0 .
\end{aligned}
$$

As they are first-order PDEs, they can be solved with standard methods (see e.g. [13]), and the solution can be expressed as

$$
\begin{aligned}
f(\mathbf{q}) & =\frac{2 q_{1}}{k+2}+a_{1} q_{1}^{-k / 2}, \quad g(\mathbf{q})=\frac{2 q_{2}}{l+2}+a_{2} q_{2}^{-l / 2}, \\
\frac{V(\mathbf{q})}{2} & =\frac{q_{1}^{k+2}}{(k+2)^{2}}+\frac{q_{2}^{l+2}}{(l+2)^{2}}+\frac{a_{1} q_{1}^{(k+2) / 2}}{k+2}+\frac{a_{2} q_{2}^{(l+2) / 2}}{l+2}
\end{aligned}
$$


for the component functions and gauge term, respectively. Clearly, as the system related to $T$ is linearizable, it admits five additional Noether symmetries, all of which possessing a zero term in $\frac{\partial}{\partial t}$ [11.

Looking now for a potential $U(t, \mathbf{q})$ that preserves the symmetry, it follows at once from the $X_{3}$-invariance that $\frac{\partial U}{\partial t}=0$, and thus the perturbed system is conservative. The invariance by $X_{1}$ implies that $U$ must satisfy the first-order differential equation (see (11)

$$
f(\mathbf{q}) \frac{\partial U}{\partial q_{1}}+g(\mathbf{q}) \frac{\partial U}{\partial q_{2}}+2 U(\mathbf{q})=0,
$$

with the functions as obtained above. A routine but tedious computation leads to the general solution

$$
U(\mathbf{q})=\frac{\Psi(u)}{q_{1}^{k}\left(2 q_{1}+a_{2}(k+2) q_{1}^{-k / 2}\right)^{2}},
$$

where

$$
u=\frac{2^{2 l^{2} /(2 l+4)^{2}} q_{2}^{(1+l) / 2}+a_{1}(l+2)}{4 q_{1}^{(k+1) / 2}+2 a_{2}(k+2)} .
$$

We skip the proof that the perturbed system $T+\varepsilon U$ possesses exactly a symmetry algebra of Noether point symmetries isomorphic to $\mathfrak{s l}(2, \mathbb{R})$.

We observe that, considering $k$ and $l$ as parameters, this approach also allows us to relate different dynamical systems with $(k, l) \neq\left(k^{\prime}, l^{\prime}\right)$ that have an isomorphic symmetry algebra, the corresponding symmetry generators being related by the parameterization of the functions $f(\mathbf{q})$ and $g(\mathbf{q})$.

\subsection{SYSTEMS WITH FIXED SYMMETRY}

Let us now consider the second possibility, namely, fixing the functions $f(\mathbf{q})$ and $g(\mathbf{q})$ in (17). To this extent, consider $f(\mathbf{q})=q_{1}^{n}, g(\mathbf{q})=q_{2}^{n}$ for simplicity, where $n \neq 0$.

In this case, the symmetry condition for $X_{1}$ leads to the system

$$
\begin{gathered}
\left(n q_{i}^{n-1}+n q_{j}^{n-1}-2\right) A_{i j}(\mathbf{q})+q_{1}^{n} \frac{\partial A_{i j}}{\partial q_{1}}+q_{2}^{n} \frac{\partial A_{i j}}{\partial q_{2}}=0 \\
q_{1}^{n} A_{11}+q_{2}^{n} A_{12}-\frac{\partial V}{\partial q_{1}}=0 \\
q_{1}^{n} A_{12}+q_{2}^{n} A_{22}-\frac{\partial V}{\partial q_{2}}=0
\end{gathered}
$$

for $(i j)=(11),(12),(22)$. It is not too difficult to see that if $A_{11}(\mathbf{q})=A_{22}(\mathbf{q}) \neq 0$ holds, then the preceding system possesses a nontrivial solution only for the values $n=0,1$. In order to find manageable solutions for arbitrary $n$, we thus assume that $A_{11}(\mathbf{q})=A_{22}(\mathbf{q})=0$. Then the system can be reduced, and for $n \neq 1$ admits the solution

$$
\begin{gathered}
A_{12}(\mathbf{q})=\left(q_{1} q_{2}\right)^{-n} \exp \left(\frac{-\left(q_{1}^{1-n}+q_{2}^{1-n}\right)}{n-1}\right), \\
V(\mathbf{q})=\exp \left(\frac{-\left(q_{1}^{1-n}+q_{2}^{1-n}\right)}{n-1}\right) .
\end{gathered}
$$

For $n=1$, we merely get $A_{12}(\mathbf{q})=1$ (the free pseudoEuclidean Lagrangian) and the gauge term $V(\mathbf{q})=$ $q_{1} q_{2}$.

Further, considering a potential $U(\mathbf{q})$ requires to solve the additional PDE

$$
q_{1}^{n} \frac{\partial U}{\partial q_{1}}+q_{1}^{n} \frac{\partial U}{\partial q_{2}}+2 U(\mathbf{q})=0
$$

that can be easily seen to provide the solution

$$
U(\mathbf{q})=\exp \left(\frac{2 q_{1}^{1-n}}{n-1}\right) \Psi\left(\frac{q_{1}^{n-1}+q_{2}^{n-1}}{\left(q_{1} q_{2}\right)^{n-1}}\right) .
$$

If we admit generalized potentials depending on $\dot{\mathbf{q}}$, the integrability condition 11 can be separated with respect to the variable $t$, because of the $X_{3}$-invariance. Skipping the details, it follows from a routine computation that the most general $U$ preserving the subalgebra $\mathfrak{s l}(2, \mathbb{R})$ in the preceding realization is given by

$$
U(\mathbf{q}, \dot{\mathbf{q}})=\exp \left(\frac{2 q_{1}^{1-n}}{n-1}\right) \Psi\left(\frac{q_{1}^{n-1}+q_{2}^{n-1}}{\left(q_{1} q_{2}\right)^{n-1}}, u_{1}, u_{2}\right),
$$

where the auxiliary variables are defined as

$$
u_{i}=\dot{q}_{i} q_{i}^{1-n} \exp \left(-\frac{2 q_{1}^{1-n}}{n-1}\right), i=1,2 .
$$

For generic choices of the function $\Psi$, the system determined by $T-\varepsilon U$ always possesses a Noether point symmetry algebra isomorphic to $\mathfrak{s l}(2, \mathbb{R})$.

\section{Time-DEPENDENT LAGRANGIANS}

As follows from Lemma 11 for conservative systems a Noether point symmetry depends at most quadratically on time. If we skip the conservative character of the system, a more wide class of possibilities is given, and (explicitly time-dependent) constants of the motion can still be guaranteed whenever an appropriate subalgebra of Noether symmetries is chosen 8 .

Let $\theta(t)$ and $\rho(t)$ be two arbitrary functions, and let $\{u(t), v(t)\}$ be an independent set of solutions of the second-order ODE

$$
\ddot{z}(t)+\rho(t) \dot{z}(t)+\theta(t) z(t)=0 .
$$

In these conditions, the function $\xi(t)=C_{1} u(t)^{2}+$ $C_{2} u(t) v(t)+C_{3} v(t)^{2}$ determines the general solution of the third-order ODE

$$
\dddot{\xi}+3 \dot{\rho} \ddot{\xi}+\left(\dot{\rho}+2 \rho^{2}+4 \theta\right) \dot{\xi}+(4 \rho \theta+2 \dot{\theta}) \xi=0 .
$$

Let us consider vector fields of the generic shape

$$
\mathbf{X}=\xi(t) \frac{\partial}{\partial t}+\frac{1}{2} \dot{\xi}(t) q_{i} \frac{\partial}{\partial q_{i}},
$$

and define $\mathbf{X}_{i}(1 \leq i \leq 3)$ as the vector field associated to the constant $C_{j}=\delta_{i}^{j}$. Computing the brackets, taking into account the constraint (25), we get the relations

$$
\begin{gathered}
{\left[\mathbf{X}_{1}, \mathbf{X}_{2}\right]=W(u, v) \mathbf{X}_{1}, \quad\left[\mathbf{X}_{1}, \mathbf{X}_{3}\right]=2 W(u, v) \mathbf{X}_{2}} \\
{\left[\mathbf{X}_{2}, \mathbf{X}_{3}\right]=W(u, v) \mathbf{X}_{3}}
\end{gathered}
$$


where $W(u, v)=u \dot{v}-\dot{u} v$ denotes the Wronskian of $\{u(t), v(t)\}$. Now, if $W(u, v)$ reduces to a constant $\lambda^{1}$ it is straightforward to verify that the vector fields $\mathbf{X}_{1}$, $\mathbf{X}_{2}$ and $\mathbf{X}_{3}$ span a Lie algebra isomorphic to $\mathfrak{s l}(2, \mathbb{R})$. For convenience, we further define the function

$$
V(\mathbf{q})=\frac{1}{4} \ddot{\xi}(t)\left(q_{1}^{2}+q_{2}^{2}\right),
$$

which shall serve as generic gauge term for the symmetry condition 111. Analyzing the symmetry condition (7), the vector fields $\mathbf{X}_{i}$ are the symmetry generators of a Noether point symmetry of a Lagrangian $L(t, \mathbf{q}, \dot{\mathbf{q}})$ whenever the first-order PDE

$$
\begin{gathered}
\frac{1}{2} \frac{\partial L}{\partial \dot{q}_{1}}\left(\ddot{\xi} q_{1}-\dot{\xi} \dot{q}_{1}\right)+\frac{1}{2} \frac{\partial L}{\partial \dot{q}_{2}}\left(\ddot{\xi} q_{2}-\dot{\xi} \dot{q}_{2}\right) \\
+\xi \frac{\partial L}{\partial t}+\frac{\dot{\xi}}{2}\left(q_{1} \frac{\partial L}{\partial q_{1}}+q_{2} \frac{\partial L}{\partial q_{2}}\right)-\frac{\dddot{\xi}}{4}\left(q_{1}^{2}+q_{2}^{2}\right) \\
-\frac{1}{2} \ddot{\xi} \dot{q}_{1} q_{1}-\frac{1}{2} \ddot{\xi} \dot{q}_{2} q_{2}+\dot{\xi} L=0
\end{gathered}
$$

is satisfied. The general solution to the latter is given by

$$
\begin{array}{r}
L(t, \mathbf{q}, \dot{\mathbf{q}})=\frac{\left(\xi \ddot{\xi}-\dot{\xi}^{2}\right)}{4 \xi^{2}}\left(q_{1}^{2}+q_{2}^{2}\right)+\frac{\dot{\xi}\left(q_{1} \dot{q}_{1}+\dot{q}_{2} q_{2}\right)}{2 \xi} \\
+\frac{1}{\xi} \Psi\left(\frac{q_{1}}{\sqrt{\xi}}, \frac{q_{2}}{\sqrt{\xi}}, \frac{\dot{\xi} q_{1}-2 \dot{q}_{1} \xi}{\sqrt{\xi}}, \frac{\dot{\xi} q_{2}-2 \dot{q}_{2} \xi}{\sqrt{\xi}}\right)
\end{array}
$$

with $\Psi$ an arbitrary function of its arguments. We observe that the previous class of Lagrangians contains, in particular, a family giving rise to oscillatory systems with a time-dependent frequency:

$$
L=\frac{1}{2}\left(\dot{q}_{1}^{2}+\dot{q}_{2}^{2}\right)+\frac{2 \ddot{\xi(t) \xi(t)-1}}{\xi(t)^{2}}\left(q_{1}^{2}+q_{2}^{2}\right) .
$$

It should be remarked that the realization of type (26) exhibits the most general form that the term in $\frac{\partial}{\partial t}$ can have, as follows at once from the following property:

Lemma 2. Let $\mathbf{X}=\xi(t, \mathbf{q}) \frac{\partial}{\partial t}+\eta_{j}(t, \mathbf{q}) \frac{\partial}{\partial q_{j}}$ be a Noether point symmetry of a regular Lagrangian $L=A_{i j}(t, \mathbf{q}) \dot{q}_{i} \dot{q}_{j}-U(t, \mathbf{q})$. Then the condition $\frac{\partial \xi}{\partial \mathbf{q}}=0$ always holds.

The proof is completely analogous to that of Lemma 1 and follows immediately from the inspection of the terms in the symmetry condition 7 having the highest power in the velocities $\dot{\mathbf{q}}$, as well as the regularity of the Lagrangian.

\section{Conclusions}

In this work we have illustrated, using functional realizations of Lie algebras based on the simple Lie algebra $\mathfrak{s l}(2, \mathbb{R})$, different possibilities to formulate a kind of inverse problem in dynamics, imposing that

\footnotetext{
${ }^{1}$ This condition is ensured whenever we set $\rho(t)=0$ in equation 24. See e.g. 14, p. 512.
}

the generators appear as Noether point symmetries of Lagrangian dynamical systems. This allows either to consider symmetry-preserving perturbations of a given system, as developed in [12, or to derive the most general Lagrangian invariant by the functional realization of the Lie algebra. The cases of conservative and dissipative systems can be treated simultaneously, considering realizations explicitly depending on timedependent functions. Albeit the examples have been restricted to the plane by simplicity, there is no obstruction to formulate the problem in arbitrary dimension. However, in order to ensure that the system is integrable [4, it is convenient to consider a realization of a symmetry algebra $\mathfrak{s l}(2, \mathbb{R}) \subset \mathfrak{g}$, so that formula (8) provides a sufficient number of independent constants of the motion. In this situation, it should be taken into account that this approach by means of Noether point symmetries in the $N$-dimensional (conservative) case is somewhat restricted, as the corresponding preserved symmetry algebras are subalgebras of the Noether point symmetry algebra of the free system defined by the kinetic term $T$ of the Lagrangian. For the Euclidean case, this corresponds to subalgebras of the Schrödinger algebra $S(N)$, and hence any semisimple Lie algebra considered in this frame must be taken as a subalgebra of $\mathfrak{s l}(2, \mathbb{R}) \oplus \mathfrak{s o}(N)$ (see e.g. [12, 15]). However, for non-Euclidean geometries, the situation may differ. As an illustrative example consider the free Lagrangian $L_{0}=\frac{1}{q_{n}^{2}}\left(\dot{q}_{1}^{2}+\cdots+\dot{q}_{n}^{2}\right)$ in the upperhalf space $U=\left\{\mathbf{q} \in \mathbb{R}^{n} \mid q^{n}>0\right\}$ endowed with the Poincaré metric

$$
d s^{2}=\frac{1}{\left(q^{n}\right)^{2}}\left(d q^{1} \otimes d x^{1}+\cdots+d q^{n} \otimes d q^{n}\right)
$$

It is well known that it admits the conformal group $S O(1, n)$ as isometry group [16, i.e., the corresponding symmetry generators are Killing vectors. It is easy to show that for any $n \geq 2$, the algebra $\mathcal{L}_{P S}$ of Lie point symmetries of the dynamical system associated to $L_{0}$ is isomorphic to the direct $\operatorname{sum} \mathfrak{s o}(1, n) \oplus \mathfrak{r}_{2}$, with $\mathfrak{r}_{2}$ the 2-dimensional affine Lie algebra. Only the Lie point symmetry $\mathbf{Y}=t \frac{\partial}{\partial t}$ fails to satisfy the condition (7), as it leads to the PDE

$$
-\frac{1}{2 q_{n}^{2}} \sum_{k=1}^{n}\left(\dot{q}_{k}^{2}+\dot{q}_{k} \frac{\partial V}{\partial q_{k}}\right)-\frac{\partial V}{\partial t},
$$

which has no solution for a gauge term $V(t, \mathbf{q})$, thus the Noether point symmetries are given by the Lie algebra $\mathcal{L}_{N S} \simeq \mathfrak{s o}(1, n) \oplus \mathbb{R}$. As a consequence, for any $n \geq 2$, the algebra $\mathcal{L}_{N S}$ of Noether point symmetries of a second-order system

$$
\begin{gathered}
\ddot{q}_{k}=\frac{2 \dot{q}_{k} \dot{q}_{n}}{q_{n}}-\frac{q_{n}^{2}}{2} \frac{\partial U}{\partial q_{k}}, \quad 1 \leq k \leq n-1, \\
\ddot{q}_{n}=\frac{\dot{q}_{n}^{2}-\dot{q}_{1}^{2}-\cdots-\dot{q}_{n-1}^{2}}{q_{n}}-\frac{q_{n}^{2}}{2} \frac{\partial U}{\partial q_{n}}
\end{gathered}
$$

with Lagrangian $L=L_{0}-U(t, \mathbf{q})$ corresponds to a subalgebra of $\mathfrak{s o}(1, n) \oplus \mathbb{R}$. These two cases indicate 
that a detailed study of the symmetry algebras of free Lagrangians corresponding to nonequivalent metrics in $N \geq 2$ dimensions would give rise to a hierarchy of Lie algebras that allows to systematize the symmetry analysis of perturbed systems. For some important types of differential equations, this approach has already provided interesting results (see [15, 17] and references therein). Further work along these lines is currently in progress.

Finally, as follows from the symmetry condition (7), a Noether point symmetry of a regular Lagrangian $L$ necessarily possesses the generic form $\mathbf{X}=\xi(t) \frac{\partial}{\partial t}+$ $\eta_{j}(t, \mathbf{q}) \frac{\partial}{\partial q_{j}}$. This suggests to study specifically realizations of Lie algebras of this type, in order to characterize those isomorphism classes of Lie algebras that appear as Noether symmetries of a system, but do not correspond to an isometry generator of the associated kinetic Lagrangian. Some developments in this direction have been proposed in [18, in connection with various geometric properties. A similar approach can be found in [15] and some previous work, where symmetries of certain types of non-autonomous systems defined in Riemann spaces have been studied, providing new insights to the geometrical interpretation of dynamical quantities. An interesting problem worthy to be analyzed in the context of the symmetry analysis of dynamical systems is the possibility of combining symmetry groups with certain of the geometric properties of the orbits determined by the solutions of a system [5], a question that has still not exhausted the possibilities of the group-theoretical approach.

\section{ACKNOWLEDGEMENTS}

The author expresses his gratitude to Prof. M. Znojil for the invitation to the AAMP XIV conference.

During the preparation of this work, the author was financially supported by the research project MTM201679422-P of the AEI/FEDER (EU).

\section{REFERENCES}

[1] E. Noether. Invariante Variationsprobleme. Nachr Ges Wiss Göttingen, Math-Phys Kl 1918:235-257, 1918.

[2] L. V. Ovsyannikov. Group Analysis of Differential Equations. Academic, New-York, 1982.

[3] L. Dresner. Application of Lie's Theory of Ordinary and Partial Differential Equations. IOP Publishing, Bristol, 1999.

[4] A. M. Perelomov. Integrable Systems of Classical Mechanics and Lie Algebras. Birkhäuser Verlag, Basel, 1990.
[5] A. S. Galiullin. Inverse Problems of Dynamics. Mir Publishers, Moscow, 1984.

[6] S. E. Jones, B. G. Vujanovic. On the inverse Lagrangian problem. Acta Mech 73:245-251, 1988. DOI:10.1007/BF01177044

[7] V. M. Gorringe, P. G. L. Leach. Lie point symmetries for systems of second-order linear differential equations. Quaest Math 11:95-117, 1988. DOI:10.1080/16073606.1988.9631946

[8] R. Campoamor-Stursberg. On certain types of point symmetries of systems of second-order ordinary differential equations. Comm Nonlinear Sci Num Simulat 19:2602-2614, 2014. DOI:10.1016/j.cnsns.2014.01.006

[9] P. G. L. Leach. Equivalence classes of second-order ordinary differential equations with only a three dimensional Lie algebra of point symmetries and linearisation. J Math Anal Appl 284:31-48, 2003. DOI:10.1016/S0022-247X(03)00147-1

[10] A. Ballesteros, et al. $N$-dimensional $s l(2)$-coalgebra spaces with non-constant curvature. Phys Letters $B$ 652:376-383, 2007. DOI:10.1016/j.physletb.2007.07.012.

[11] G. Gubbioti, M. C. Nucci. Are all classical superintegrable systems in two-dimensional space linearizable? J Math Phys 58:012902, 2017. DOI:10.1063/1.4974264

[12] R. Campoamor-Stursberg. Perturbations of Lagrangian systems based on the preservation of subalgebras of Noether symmetries. Acta Mech 227:1941-1956, 2016. DOI:10.1007/s0070

[13] E. Kamke. Differentialgleichungen. Lösungsmethoden und Lösungen. Band II. Akademische Verlagsgesellschaft, Leipzig, 1962.

[14] E. Kamke. Differentialgleichungen. Lösungsmethoden und Lösungen. Band I. Akademische Verlagsgesellschaft, Leipzig, 1962.

[15] L. Karpathopoulos, A. Paliathanasis, M. Tsamparlis. Lie and Noether point symmetries for a class of nonautonomous dynamical systems. J Math Phys 58:082901, 2017. DOI:10.1063/1.4998715.

[16] Y. Fuji, K. Yamagishi. Killing spinors on spheres and hyperbolic manifolds. J Math Phys 27:979-981, 1986. DOI:10.1063/1.527118

[17] M. Tsamparlis, A. Paliathanasis, A. Qadir. Noether symmetries and isometries of the minimal surface Lagrangian under constant volume in a Riemannian space. Int J Geom Methods Mod Phys 12:1550033, 2015.

[18] R. Campoamor-Stursberg. An alternative approach to systems of second-order ordinary differential equations with maximal symmetry. Realizations of $\mathfrak{s l}(n+2, \mathbb{R})$ by special functions. Comm Nonlinear Sci Num Simulat 37:200-211, 2016. DOI:10.1016/j.cnsns.2016.01.015. 